\title{
Acute Visual Loss Secondary to Arnold Chiari Type I Malformation Completely Resolving After Decompressive Posterior Fossa Surgery
}

This article was published in the following Dove Press journal: International Medical Case Reports Journal

\author{
Chrysoula Florou' \\ Konstantinos Andreanos 1 \\ Nikos Georgakoulias ${ }^{2}$ \\ Edroulfo Espinosa ${ }^{2}$ \\ Evangelia Papakonstantinou' \\ Ilias Georgalas $\mathbb{D}^{\prime}$ \\ Tryfon Rotsos (iD) \\ 'Department of Ophthalmology, \\ University of Athens, G Gennimatas \\ General Hospital, Athens, Greece; \\ ${ }^{2}$ Neurosurgery Department, \\ G Gennimatas General Hospital, Athens, \\ Greece
}

Correspondence: Tryfon Rotsos Department of Ophthalmology,

University of Athens, G Gennimatas

General Hospital, Alkmanos 15 Street,

Athens II528, Greece

Tel +306974727279

Email tryfonrotsos@hotmail.com

\begin{abstract}
We describe the case of a 22-year-old woman of southeast-Asian origin, presenting with unilateral sudden visual loss after a self-healing hearing loss a week before. Ophthalmological examination showed visual acuity of light perception in the left eye, mild RAPD, normal ocular motility and an elevated optic disc with indistinct margins. Neurological examination showed no acute pathology and brain CT-MRI imaging revealed a small-almost subclinical-herniation of the cerebellar tonsils. As investigation eliminated every other infectious or inflammatory cause of papillitis, neurosurgical intervention was proposed. The patient underwent an uncomplicated occipital craniotomy with posterior fossa decompression and had a favorable revolution with regression of papilledema and a fully recovering visual acuity that reached 20/20. Chiari malformation type I refers to an abnormality of the posterior fossa that has a smaller volume than normal, leading to the herniation of cerebellar tonsils, at least $5 \mathrm{~mm}$ below the foramen magnum. The occurrence of papilledema associated with Chiari malformation type 1 is rare. Chiari malformation has, until today, mainly been studied among children populations, usually with a poor visual acuity recovery. The originality of our case report consists in the description of an adult patient case showing unilateral, unusual ophthalmological findings and complete recovery after surgical treatment.
\end{abstract}

Keywords: Arnold Chiari malformation type I, herniation of cerebellar tonsils, elevated optic disc, papilledema

\section{Introduction}

Chiari malformations, types I-IV, refer to a spectrum of hindbrain abnormalities leading to herniation of cerebellar tonsils and affecting the structural relationships between the cerebellum, brainstem, upper cervical cord, and the bony cranial base. ${ }^{1}$

Acquired type 1 Chiari malformation (CM-I) can occur when there is a significant cerebral spinal fluid (CSF) pressure gradient across the craniocervical junction, ie, trauma, scoliosis, CSF leakage or lumboperitoneal shunts can produce negative downward pressure gradients leading to the development of the disease. In addition, conditions associated with raised intracranial pressure, such as hydrocephalus and Idiopathic Intracranial Hypertension (IIH), can promote downward pressure gradient. $^{2}$

Routinely, the diagnosis of CM-I is made when an isolated cerebellar tonsillar descent, of at least $5 \mathrm{~mm}$ below the foramen magnum, is identified. It is found incidentally in $14-30 \%$ of the patients; however, patients do not present any 
symptoms until later in the adolescent or adult years of life. Symptoms develop as a result of compression of medulla and upper spinal cord, compression of cerebellum and disruption of CSF flow through foramen magnum. ${ }^{3}$

Most common symptoms include headache, ${ }^{5}$ and neck pain exacerbated by cough and Valsalva maneuver, ataxia, dysmetria, disequilibrium and hydrocephalus. Visual symptoms are present in up to $80 \%$ of the patients and include nystagmus, strabismus and diplopia. ${ }^{4}$ Eye movement deficits in the context of typical symptomatic presentation are critical clinical markers for the diagnosis of Chiari malformation. ${ }^{3}$

Herein we describe a case of a patient with Arnold Chiari malformation type I with acute visual acuity deterioration as her main presenting ophthalmic symptom.

\section{Case Presentation}

A 22-years-old woman of southeast-asian origin with no prior significant ophthalmological or family history presented to the ophthalmological emergencies complaining of acute, painless, unilateral, visual loss of the left eye. A week before visual symptoms commence, she reported transient unilateral hearing loss that resolved without treatment.

On initial examination the right eye had a visual acuity of 20/20 and no pathological findings. The left eye had visual acuity of light perception and a mild relative afferent pupillary defect (RAPD). Ocular motility was normal with no sign of strabismus or nystagmus. Slit-lamp examination showed a clear cornea and a quiet and deep anterior chamber in both eyes. Fundus examination of the left eye revealed an elevated optic disc with superior indistinct margins (Figure 1A). The patient was admitted in our clinic for further ophthalmological and neurological investigation.

Fluorescence (FA) - and Indocyanine Green Angiography were performed. Both examinations were unremarkable in the right eye. In the left eye however FA showed staining of the lower margin of the optic disc (Figure 2A and B). Visual field examination confirmed significant amaurosis on the left eye (Figure 2C).

A thorough workup was ordered in order to identify the cause of visual loss. Chest and hip x-rays were negative, C-reactive protein (CRP) and erythrocyte sedimentation rate (ESR) levels were normal and a further series of negative diagnostics excluded all possible immunologicalinflammatory or infectious causes. Neurological examination showed no acute pathology. Brain CT imaging was negative for hemorrhage and revealed a left, small herniation of the cerebellar tonsils characterized as "of no clinical significance". Intracranial pressure (ICP) was normal at the level of $10 \mathrm{mmHg}$.

After a neurosurgical evaluation including a brain MRI (Figure 1C) which confirmed the CT findings the decision for an immediate surgical treatment was made. Under endotracheal anesthesia, the patient was positioned supine. The head was clumped in the Mayfield. A midline incision from the inion to the level of $\mathrm{C} 5$, a posterior fossa decompression and laminotomy of $\mathrm{C} 1$ was performed. The dura was opened in $\mathrm{Y}$ fashion revealing the arachnoid membrane which was dissected revealing the cisterna magna. Afterwards, a cerebellar tonsillectomy was performed preserving both posterior inferior cerebellar artery (PICA) (Figure 1D). The patient showed a quick and unremarkable postoperative recovery.

An impressive improvement was noted during the onemonth post-op examination. Visual acuity was $20 / 20 \mathrm{sc}$ in both eyes and the optic disc at the left eye showed a minimal residual marginal haziness (Figure 1B). Retinal nerve fibre layer analysis (RNFL) more was also noticeably improved.

\section{Discussion}

This patient has proved to be an interesting case, as she firstly did not fit the category I Chiari syndrome due to the mild findings of brain $\mathrm{CT}$ and the atypical clinical manifestations.

Headache, the most common symptom in CM-I, was absent, though the patient mentioned mild neck pain during coughing after a detailed medical history was taken. Patients usually describe a dull occipital pain, accompanied by throbbing, nausea and vomiting, that is exacerbated by coughing, lack of sleep and alcohol consumption. ${ }^{6}$

Both strabismus and diplopia were also absent. CM-I very often presents with strabismus, typically esotropia, pathophysiologically explained, for example, as a result of coexisting sixth nerve palsy. Acquired comitant strabismus, divergence insufficiency or skew deviation are also common first manifestations in patients with minimal cerebellar tonsil herniation. ${ }^{4}$

Although the most common presentation of CM-I is with occipital headache and neuro-ophthalmological findings, the association of audio-vestibular symptoms in such patients is not rare.

Vertigo, a sudden hearing loss, unsteadiness, dizziness, nystagmus, could also be suggestive symptoms. In fact, 


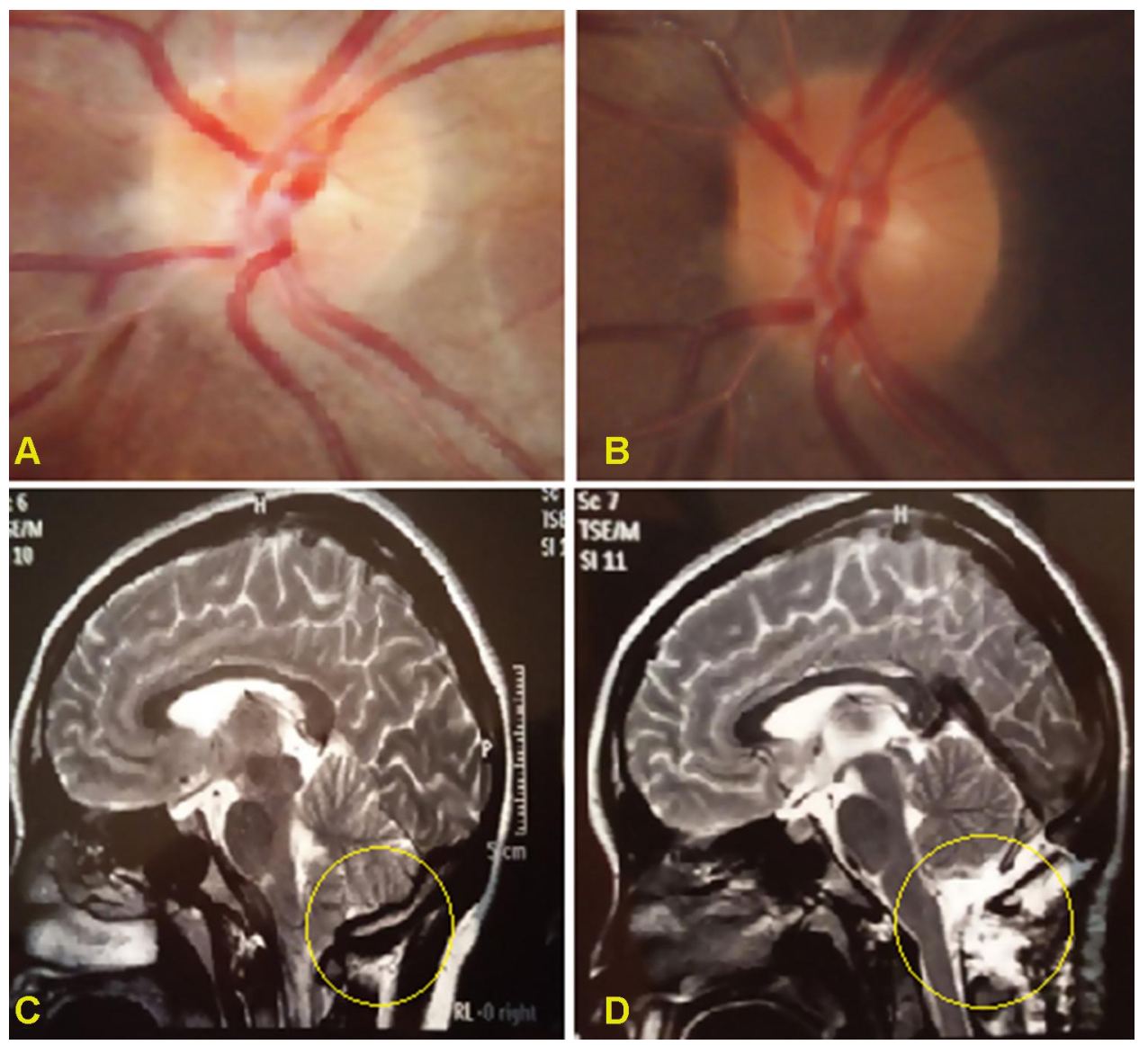

Figure I (A) Fundus photo on presentation: elevated left optic disc with indistinct margins. (B) Fundus photo I month after posterior fossa decompression with duraplasty. Regression of papilledema. (C) Pre-operative MRI: Herniation of the cerebellar tonsils below the foramen magnum. (D) MRI after posterior fossa decompression with duraplasty and cerebellar tonsillectomy.

the development of such clinical signs and their intensity are more related to the presence of an anomalous spinal fluid circulation pattern that the absolute degree of cerebellar tonsil descent. ${ }^{7}$

Papilloedema, occasionally followed by ischemia and eventual visual impairment or loss, is a rare but important manifestation of Chiari I malformation, with a clinical presentation often similar to that of idiopathic intracranial hypertension. The interesting aspect in our case is that our patient presented with a unilateral optic disc findings.

The pathogenesis is not well known, but potential mechanisms causing papilloedema include a mechanical and an ischemic theory. Mechanical theory suggests that elevated intracranial pressure (ICP) causes direct compression of axons, the high ICP compresses the optic nerve $(\mathrm{ON})$ at its junction with the globe and distends its prelaminar axons as their axoplasm piles up at the scleral lamina. On the other hand, ischemic theory supports that severe dilation of the distal optic subarachnoid space can lead to compression of regional neural and vascular structures, compromising vascular flow. If the subarachnoid pressure rises, the ciliary circulation becomes compressed and the arterial blood supply to the laminar $\mathrm{ON}$ is reduced. The ON axons then become distended as chronic ischemia interferes with metabolic axoplasmic flow. ${ }^{8,9}$

A structural abnormality in the posterior fossa in subjects with CM-I can lead to malignant increases in cerebrospinal fluid pressure. Such deficit can present with acute onset of visual loss and malignant papilledema, requiring emergent cerebrospinal fluid shunt procedures. Decompressive operations aim to increase the postoperative posterior cranial fossa/cisterna magna volume, which is associated with a greater likelihood of clinical improvements of headache and tonsillar descent in patients with CM-I. In such cases, both posterior fossa decompression with or without duraplasty have proven to be equally effective. ${ }^{10}$

Though increased ICP seems to be a common mechanism in the development of a papilloedema, our case seems to be better explained through the mechanical traction of the left optic nerve caused by the ipsilateral herniation of 

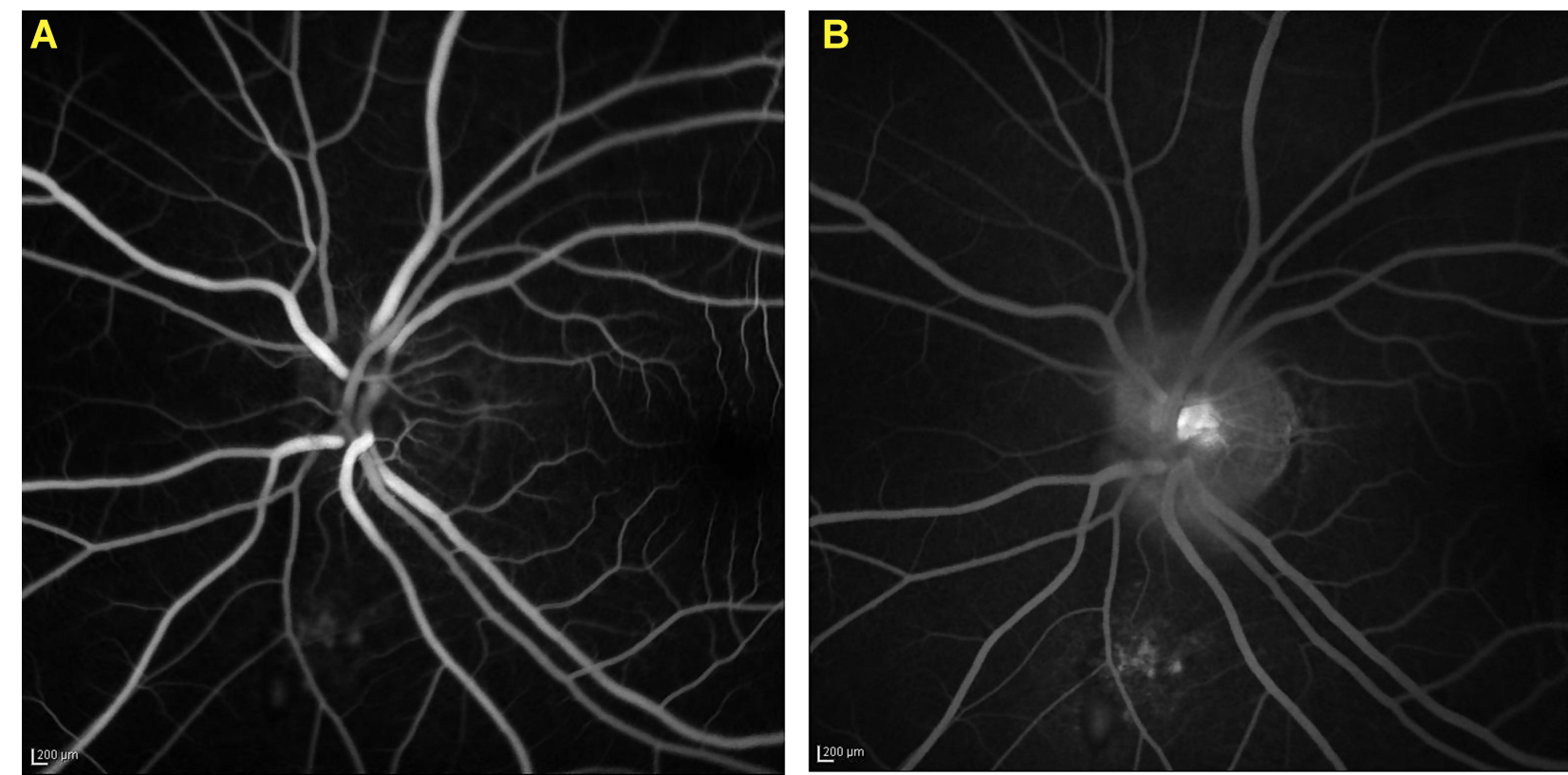

Central 30-2 Threshold Test

Fixation Monitor: Gaze/Blind Spot

Stimulus: V. White

Background: 31.5 ASB

Fixation Losses: 2/25

False POS Errors: $0 / 17$

False NEG Errors: $1 / 6$

Test Duration: 15:27

Fovea: OFF

C

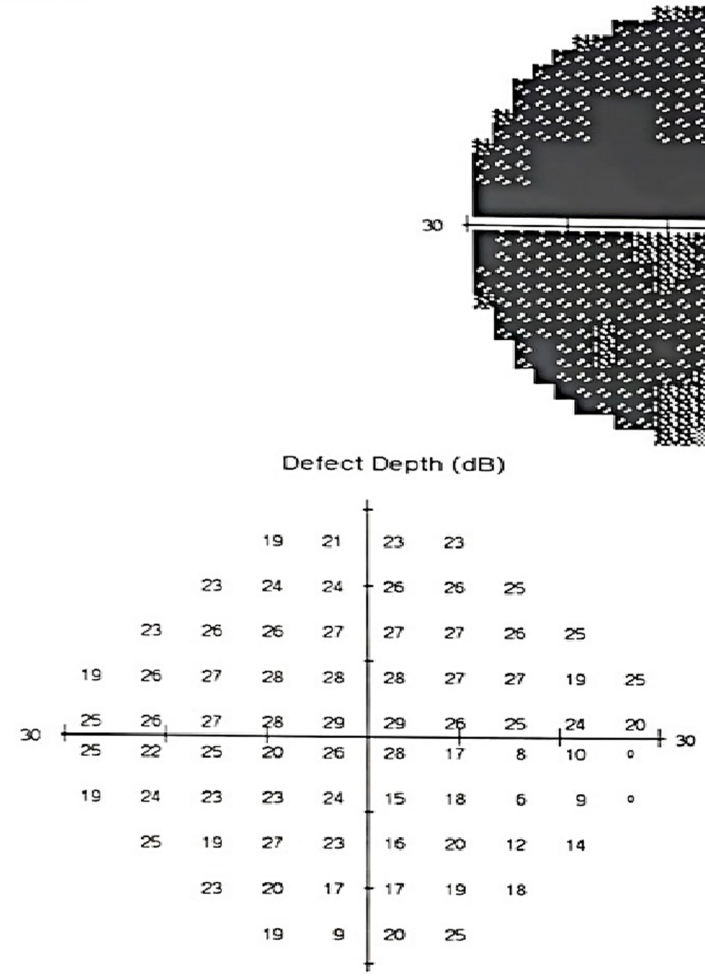

Pupil Diameter:

Visual Acuity:

$\mathrm{RX}$ : DS DC $x$
Date: 03-01-2017

Time: 11:43

Age: 23

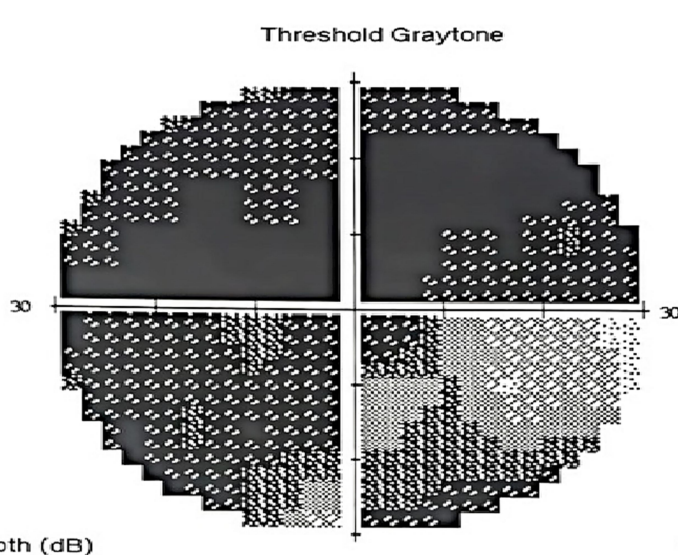

Threshold (dB)

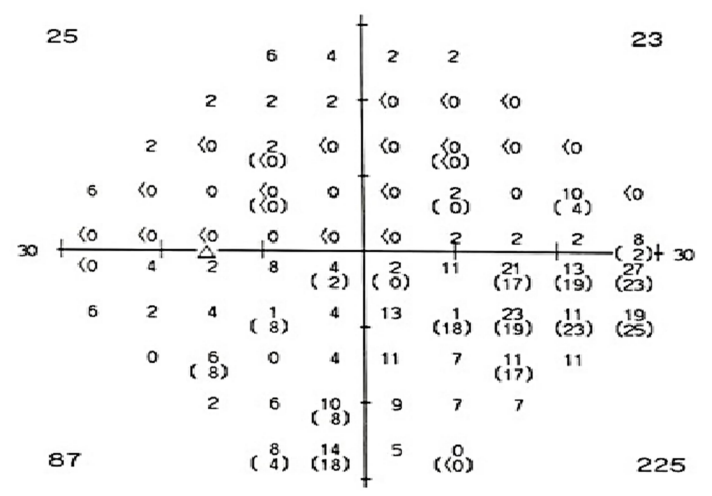

Figure 2 (A and B) Early and late venous FA phases showing staining of the lower margin of the left optic disc. (C) Left visual field examination showing severe, almost amaurotic, defects. 
the cerebellar tonsils, as ICP was normal in our case. A possible mechanism could involve compression of the Posterior Cerebral arteries against the Tentorium and therefore a mechanical constrict which decreases the blood flow to the occipital lobes.

Additional aspects that support our theory were the facts that the papillary findings were unilateral, ipsilateral to the CT pathology and that the patient showed no typical papilloedema but a unilateral elevated optic disc with indistinct margins.

Optic neuritis is another condition that could explain the acute vision loss, and has a natural history of spontaneous recovery in a few weeks. However, MRI findings were not supportive. The patient did not receive steroid treatment at any time near the surgery or any other medical treatment that could improve her vision, in case of optic neuritis was the possible diagnosis. Furthermore, visual recovery was noted from the first post-operative day further supporting the theory of vision loss due to mechanical traction.

To recapitulate, we present a case of CM-I with atypical clinical signs such as severely decreased visual acuity and unilateral optic disc edema as her main symptoms. She underwent posterior fossa decompression with duraplasty with a very favorable outcome, full recovery of visual acuity, reaching 20/20, and regression of papilledema 1 month postoperatively.

\section{Ethical Approval}

This article does not contain any studies with human participants or animals performed by any of the authors. Ethical and clinical approval for this publication was granted from the Ethical and Medical Committee of Hospital G.Genimmatas.

\section{Informed Consent}

Informed consent was obtained from all individual participants included in the study. Written informed consent has been provided by the patient to have the case details and any accompanying images published.

\section{Funding}

There was no funding or grants for this study.

\section{Disclosure}

The authors report no conflicts of interest in this work.

\section{References}

1. Vidmer S, Sergio C, Veronica S, et al. The neurophysiological balance in Chiari type 1 malformation (CM1), tethered cord and related syndromes. Neurol Sci. 2011;32(Supplement 3):311-316. doi:10.1007/s10072-011-0692-3

2. Doberstein CA, Torabi R, Klinge PM. Current concepts in the pathogenesis, diagnosis, and management of type I Chiari Malformations. Med J. 2017;100(6):47-49.

3. Imane M, Asmae M, Toufik R, Rachid S. Papillary oedema revealing Arnold Chiari malformation type 1: about a case. Pan Afr Med J. 2016;24:293

4. Shaikh AG, Ghasia FF. Neuro-ophthalmology of type 1 Chiari malformation. Expert Rev Ophthalmol. 2015;10(4):351-357. doi:10.1586/17469899.2015.1057505

5. Abu-Arafeh I, Campbell E. Headache, Chiari malformation type 1 and treatment options. Arch Dis Child. 2017;102(3):210-211. doi:10.1136/archdischild-2016-312137

6. Siavoshi S, Dougherty C, Ailani J, Yadwadkar K, Berkowitz F. An unusual case of post-traumatic headache complicated by intracranial hypotension. Brain Sci. 2016;7(1):3. doi:10.3390/brainsci7010003

7. Guerra Jiménez G, Mazón Gutiérrez Á, Marco de Lucas E, Valle San Román N, Martín Laez R, Morales Angulo C. Audio-vestibular signs and symptoms in Chiari malformation type I. Case series and literature review. Acta Otorrinolaringol Esp. 2015;66(1):28-35. doi:10.1016/j.otorri.2014.05.002

8. Zhang JC, Bakir B, Lee A, Yalamanchili SS. Papilloedema due to Chiari I malformation. BMJ Case Rep. 2011;16:2011.

9. Passi N, Degnan AJ, Levy LM. MR imaging of papilledema and visual pathways: effects of increased intracranial pressure and pathophysiologic mechanisms. AJNR Am J Neuroradiol. 2013;34 (5):919-924. doi:10.3174/ajnr.A3022

10. Yuh WT, Kim CH, Chung CK, Kim HJ, Jahng TA, Park SB. Surgical outcome of adult idiopathic Chiari malformation type 1. J Korean Neurosurg Soc. 2016;59(5):512-517. doi:10.3340/jkns.2016.59.5.512

\section{Publish your work in this journal}

The International Medical Case Reports Journal is an international, peer-reviewed open-access journal publishing original case reports from all medical specialties. Previously unpublished medical posters are also accepted relating to any area of clinical or preclinical science. Submissions should not normally exceed 2,000 words or 4 published pages including figures, diagrams and references. The manuscript management system is completely online and includes a very quick and fair peer-review system, which is all easy to use. Visit http://www.dovepress.com/testimonials.php to read real quotes from published authors. 